\title{
Culture of urinary sediment for the diagnosis of gonorrhoea in women
}

\author{
THOMAS A. CHAPEL AND MIKE SMELTZER \\ Venereal Disease Clinic, Columbus, Ohio, U.S.A.
}

Gonorrhoea is currently the most commonly reported communicable disease in the United States. The epidemic stems in part from our relative inability to identify the disease in asymptomatic women. Consequently, control programmes are designed to detect gonorrhoea in the female population through widespread screening by culture of anogenital sites on selective media. However, the logistics of this screening method limits the number of women that can be tested. A simple but reliable screening tool would aid control efforts. This paper evaluates the culturing of urinary sediment on selective media for the diagnosis of gonorrhoea in women. It also discusses the symptoms of women with and without gonococcal infections who were interviewed in a V.D. clinic.

\section{Material and methods}

Study population and preparation of specimens

Endocervical and anal canal cultures and cultures of urinary sediment were obtained from 1,001 consecutive women seen at the Venereal Disease Clinic in Columbus, Ohio, from September 9, 1972, to April 27, 1973.

All patients were interviewed for history of urogenital symptoms. Before examination each patient voided a urine specimen in a clean but nonsterile container. No attempt was made to obtain a 'clean-catch' urine.

Within $1 \mathrm{hr}$ of collection a $15 \mathrm{ml}$. aliquot of urine was centrifuged at $4,000 \times \mathrm{G}$ for $15 \mathrm{~min}$. The supernatant fluid was carefully decanted and, with a $2 \mathrm{~mm}$. platinum bacteriological loop, three or four loopfuls of sediment were streaked on a Thayer-Martin plate. The plate was then incubated at $35^{\circ} \mathrm{C}$. in a partial carbon dioxide atmosphere obtained by candle extinction in a jar.

Cultures were obtained from the endocervix and anal canal by sterile, plain, cotton-wool tipped swabs. The specimens were immediately processed as outlined for the urinary sediment cultures.

Received for publication June 25, 1974

Address for reprints: Dr. T. A. Chapel, M.D., Wayne State University School of Medicine, 540 East Canfield Avenue, Detroit, Michigan 48201, U.S.A.

\section{Isolation and identification of Neisseria gonorrhoeae}

All Thayer-Martin plates were examined after $24 \mathrm{hrs}$ incubation and, if negative, at $48 \mathrm{hrs}$. $N$. gonorrhoeae were identified by typical colonial morphology, oxidase reaction, and Gram stain. Presumptively positive cultures were tested for fermentation of 1 per cent. glucose and maltose in cystine trypticase agar medium (Difco) and also by the specific staining reaction with immunofluorescent gonococcal antiserum (Difco).

\section{Results}

$N$. gonorrhoeae were isolated from 285 (28.5 per cent.) of the 1,001 patients examined. Table I shows the culture results by site. The endocervix was positive in $252(25.2$ per cent.) of the women, the anal canal in 69 ( 6.9 per cent.), and the urinary sediment in 206 (20.6 per cent.). Urinary sediment was concomitantly positive with the endocervix in 177 (17.7 per cent.) of the women.

\section{TABLE I Sites of recovery of gonococcus}

\begin{tabular}{lr}
\hline Sites & No. of cases \\
\cline { 1 - 2 } Positive urine, endocervix, and anal canal & 48 \\
Positive urine and endocervix only & 129 \\
Positive urine and anal canal only & 4 \\
Positive urine only & 25 \\
Positive endocervix and anal canal only & 62 \\
Positive endocervix only & 4 \\
Positive anal canal only & 285 \\
\hline Total & \\
\hline
\end{tabular}

The urine was positive in 206 ( 73.3 per cent.) of 281 specimens that were positive from either the endocervix or urinary sediment; the endocervix was positive in 252 ( $89 \cdot 7$ per cent.) of these 281 specimens.

Symptoms in patients with and without gonorrhoeal infections

All patients were asked about urogenital symptoms. Approximately 40 per cent. of the 1,001 patients had more than one complaint. Tabulation by major symptoms for both culturally negative and culturally positive women is shown in Table II. Urogenital 
symptoms were present in $55 \cdot 1$ per cent. of women with negative or overgrown cultures and 64.6 per cent. of women with $N$. gonorrhoeae isolated from one or more sites. The difference is statistically significant by $\chi^{2}$ analysis $(P<\cdot 01)$. Symptoms were mentioned by $65 \cdot 1$ per cent. of all patients with positive urine examinations and 64.2 per cent. of all patients with positive endocervix examinations.

\section{Correlation of positive cultures with symptoms}

Symptoms described by women with positive and with negative cultures are compared in Table II. Vaginal discharge was the most common major complaint. The likelihood of a positive culture was highest when either dysuria or pelvic pain was the major complaint. The percentage of women with positive cultures at one or more sites varied significantly $(P<0.005)$ for the different categories of complaints (i.e. asymptomatic, discharge, dysuria, itch, pelvic pain, miscellaneous). Dysuria was more common among patients with a positive urine culture than among patients with only positive anogenital cultures, $21 \cdot 8 v .7 .6$ per cent. Only 11.7 per cent. of the patients negative for gonorrhoea had dysuria.

\section{Overgrowth}

Overgrowth was observed more frequently in urinary than in anogenital cultures. Only 23 of $1,001(2 \cdot 3$ per cent.) endocervical cultures were overgrown, while 51 of 1,001 (5.1 per cent.) anal canal and 82 of 1,001 (8.2 per cent.) urine cultures were overgrown. In no case was an overgrown endocervical specimen positive by either anal canal or urine, but 15.7 per cent. of the 51 overgrown anal canal and $17 \cdot 1$ per cent. of the 82 overgrown urine cultures were positive on one or both of the other specimens.

\section{Discussion}

The majority of women seen in the V.D. clinic had urogenital symptoms and these symptoms were significantly more common in women infected with gonorrhoea than in non-infected women. Schroeter and Lucas (1962) have stated that 80 per cent. of women with gonorrhoea are asymptomatic. In our study, however, only 35.4 per cent. of women with gonorrhoea were symptom free.

Several papers have dealt with recovery of the gonococcus from urine specimens in men. Taggart (1955) visually inspected fresh urine specimens treated with 10 per cent. acetic acid and, if shreds or pus were observed, the specimen was considered presumptively positive for gonorrhoea. Sediment was also examined microscopically for Gramnegative intracellular diplococci. Results from both methods were compared with Gram-stained smears or cultures of urethral exudate. In symptomatic men, macroscopical examination agreed with positive urethral smears or cultures in 81 per cent. and microscopical analysis in $\mathbf{7 6 . 5}$ per cent.

Ch'in (1938) studied the viability of gonococci in the urine of male patients with acute and chronic urethritis. He cultured urinary sediment and uncentrifuged urine. The average period of gonococcal viability in sediment kept at room temperature was $27 \mathrm{hrs}$. Gonococci in uncentrifuged urine disappeared much earlier and overgrowth was more frequent.

Allison, Charles, and Carpenter (1942) cultured urine from 127 males with urethritis. Gonococci were identified in 86 urines and 102 Gram-stained smears of urethral exudate. Urine sediment yielded viable gonococci after $3 \mathrm{hrs}$ at room temperature. After 12 to $15 \mathrm{hrs}$ the recovery rate dropped to 84 per cent.; at $24 \mathrm{hrs}$ to 41 per cent.; and at $48 \mathrm{hrs}$ to only 2 per cent. More positive cultures were isolated from urine sediment than from uncentrifuged urine. Refrigeration improved recovery of gonococci from urines held for $24 \mathrm{hrs}$ before culturing. Urinary $\mathrm{pH}$ on the other hand seemed to have little, if any, influence on the viability of the gonococcus.

Moore, Pittard, Mosca, and O'Brien (1973) evaluated 392 men for gonorrhoea using Gramstained smears and cultures of urinary sediment and urethral discharge. A total of 238 cases of gonorrhoea were identified by culture of the discharge and 224

TABLE II Major complaint of patients with negative and positive cultures for Neisseria gonorrhoeae

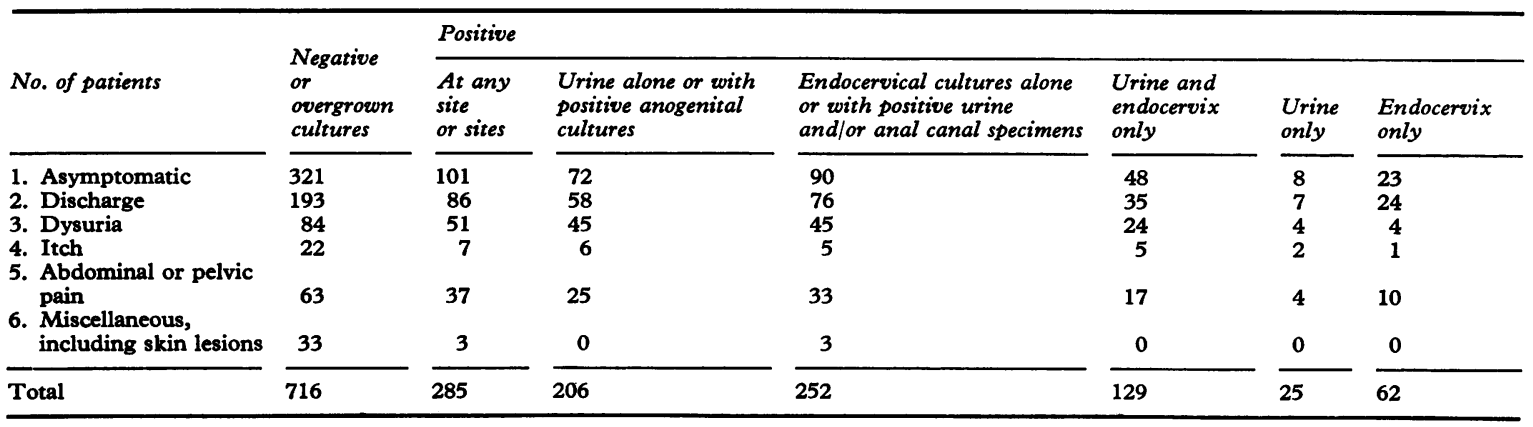


(94.1 per cent.) by culture of the urinary sediment. The authors concluded that culture of urinary sediment was a valuable tool in screening men for gonorrhoea.

Recovery of Neisseria gonorrhoeae from the urinary sediment of women presumably resulted from a gonococcal urethritis, although some specimens may have been contaminated by gonococci in vaginal secretions. The endocervix is generally felt to be the primary focus of gonorrhoea infection in the female, but urethral involvement is common. Schmale, Martin, and Domesčik (1969) found that 91.4 per cent. of women with gonorrhoea had positive endocervical cultures and 74.8 per cent. were positive by urethral culture. These data correlate well with our own finding that gonococci were cultured from the urine in 72.3 per cent. and from the endocervix in 88.4 per cent. of patients with gonorrhoea. It is highly unlikely that positive urine cultures represented bladder or kidney infection, though documented cases have been reported.

Acute gonococcal urethritis in females may be associated with symptoms of dysuria and frequency and urgency of urination. However, because of the shortness of the female urethra, its predominantly squamous cell epithelium and paucity of deep glands, these symptoms generally subside rapidly. Our data showed an increased likelihood of a positive urine or endocervical culture in women with these complaints.

Urinary symptoms in women with cultures negative for gonorrhoea may have been manifestations of the urethral syndrome or of cystitis due to microorganisms inhibited by the Thayer-Martin media. The urethral syndrome of dysuria and frequency of micturition with or without significant bacteriuria is common in sexually active women. The syndrome is associated with urethral trauma, such as sexual intercourse, which predisposes to bacterial infection.

Neisseria gonorrhoeae was recovered on endocervical culture from 88.4 per cent. of patients with gonorrhoea detected by any method, while cultures of urinary sediment were positive in 72.3 per cent. and the combination of endocervical and anal canal cultures in 91.2 per cent. The data re-emphasize the greater reliability of anogenital culturing for routine diagnosis of gonorrhoea in women. However, the logistics of anogenital culturing tends to limit the size of screening programmes. The decreased sensitivity of urinary sediment cultures is partially offset by the ease with which specimens are obtained. Therefore, culture of urinary sediment could provide a simple and reasonably effective tool for screening large numbers of women for gonorrhoea, particularly in the absence of resources for endocervical cultures.

\section{Summary}

1,001 consecutive women seen in a V.D. clinic were screened for gonorrhoea by cultures of urinary sediment, the endocervix, and anal canal. Recovery of Neisseria gonorrhoeae on culture of urinary sediment was 81.7 per cent. as productive as culture of the endocervix, and 79.2 per cent. compared to endocervical and anal canal culturing.

Urogenital symptoms were present in 55.1 per cent. of women with negative cultures and 64.6 per cent. of those with gonorrhoea. A complaint of dysuria increased the likelihood of a positive urinary sediment or endocervical culture.

Culture of urinary sediment is less sensitive than culture of the endocervix. However, the ease with which specimens are obtained and the relative simplicity of the technique could make this method an acceptable tool for screening large numbers of women for gonorrhoea.

\section{References}

Allison, S. D., Charles, R., and Carpenter, C. M. (1942) Vener. Dis. Inform., 23, 283

CH'IN, T. L. (1938) Chin. med. f., 53, 227

Moore, G., Pittard, W. B., Mosca, N., and O'Brien, W. M. (1973) F. Amer. med. Ass., 224, 1499

Schmale, J. D., Martin, J. E., and Domesčik, G. (1969) Ibid., 210, 312

SCHROETER, A. L., and LuCAS, J. B. (1972) Obstet. and Gynec., 39, 274

Taggart, S. R. (1955) Publ. Hlth Rep. (Wash.), 70, 245

La culture du sédiment urinaire dans le diagnostic de la gonococcie féminine

\section{SOMMAIRE}

Les cultures du sédiment urinaire, de l'endocol et du canal anal furent utilisées pour la détection systématique de la gonococcie sur un total de 1001 femmes vues consécutivement dans une clinique vénéréologique. Pour déceler la gonococcie, la culture du sédiment urinaire fut efficace à 81,7 pour cent en comparaison avec la culture de l'endocol et à 79,2 pour cent en comparaison avec les cultures jumelées de l'endocol et du canal anal.

Des symptômes uro-génitaux furent notés chez 55,1 des femmes à culture négative et chez 64,6 pour cent des gonococciques. La probabilité de la positivité de la culture du sédiment urinaire ou de l'endocol fut accrue en cas de dysurie.

La culture du sédiment urinaire est moins sensible que la culture de l'endocol. Cependant, la facilité d'obtenir des échantillons et la simplicité relative de la technique peut faire de cette méthode un moyen acceptable pour la recherche systématique de la gonococcie lorsque celle-ci concerne de grands nombres de femmes. 